\title{
PARTICIPACIÓN DE LAS MUJERES INDÍGENAS DE RAMA CAY, RAAS, EN LOS ESPACIOS DE TOMA DE DECISIÓN
}

Narda Wilson Blandford ${ }^{[1]}$

\section{Resumen}

El estudio ha versado sobre la participación de las mujeres indígenas rama de la comunidad de Rama Cay en los espacios de la toma de decisiones.

El objetivo planteado fue comprender la lógica estructural de esta dinámica. Se implementó la metodología cualitativa y se enfocó en la Teoría Crítica, particularmente la que se inscribe en el conocimiento de las reglas implícitas que generan actuación e interpretación. Técnicamente se describe e interpreta la actuación de las mujeres ramas en determinados contextos políticos, lo cual implica necesariamente adentrarse en las esferas del poder. El estudio presenta la situación de la mujer indígena rama de la comunidad de Rama Cay en los espacios de toma de decisiones, las limitantes y los desafíos a fin de lograr una mayor integración y participación cualitativa en estos ámbitos.

Palabras Clave: Participación, mujer indígena y toma de decisión, Costa Caribe nicaragüense.

\section{Summary}

The study is focused on the participation of Rama's indigenous women from the community of Rama Cay in decision-making spaces.

The objective was to understand the structural logic of this dynamic. Qualitative methodology was implemented and it focused on the critical theory, particularly those that are inscribed in the knowledge of the implicit rules that generate action and interpretation. Technically it describes and interprets the performance of the Rama's women in certain political contexts, which necessarily involves getting inside the spheres of power. The study presents the situation of indigenous women from Rama Cay community in decision-making spaces, as well as the constraints and challenges to achieve greater integration and qualitative participation in these areas.

Keywords: Participation, indigenous women and decision-making, Caribbean Coast of Nicaragua.

${ }^{[1]}$ Máster en Desarrollo Con Identidad Territorialidad Gobernabilidad Manejo de Bosque.
Investigadora nardawilson@yahoo.com 


\section{Introducción}

La participación ciudadana es el espacio de interacción social, de intervención directa entre mujeres y hombres en las tomas de decisiones como parte de la democracia. Pero, esencialmente es la participación para afectar la toma de decisiones, ejecución y seguimiento a las decisiones públicas.

En este sentido, la participación de la mujer indígena es un factor importante, ya que les da la oportunidad de presentar sus demandas en las diferentes esferas políticas a fin de lograr la reivindicación de sus derechos y aportar con sus ideas, conocimientos y experiencias para el desarrollo de su comunidad.

Las comunidades indígenas y específicamente las mujeres enfrentan problemas de violencia, discriminación y desigualdad. Obligadas por lo tanto, a buscar su integración en los espacios de toma de decisión a nivel comunal, municipal, regional y nacional a fin de presentar sus demandas por la reivindicación de sus derechos de igualdad, respeto y las oportunidades de aportar efectivamente al desarrollo de sus comunidades.

En la comunidad indígena de Rama Cay se continúa observando una mayor participación de los hombres en los espacios de toma de decisión en el ámbito comunal, territorial y regional.

A través de esta investigación se pretende analizar cómo es la participación de las mujeres indígenas de Rama Cay en los ámbitos de poder, en qué contribuyen al desarrollo de su comunidad y cuáles han sido los factores que han incidido en su participación en los espacios de toma de decisiones. Así mismo, se proporciona información para un mejor entendimiento de las relaciones de desigualdad, de discriminación y la limitada participación de la mujer indígena de Rama Cay.

La investigación se contextualiza en los debates relacionados a participación, la teoría de género en el marco de la Teoría Crítica presentada por Mejía y Sandoval (1988).

\section{Revisión de Literatura}

La participación entendida como un espacio de interacción social, en la toma de decisiones como parte de la democracia. De acuerdo a Oakley (1991) la participación ciudadana, significa diferentes cosas para diferentes personas, pero es la participación para afectar la toma de decisiones, ejecución y seguimiento de las políticas públicas.

Según la URACCAN (2009), la participación ciudadana la constituyen los elementos y mecanismos que impulsan el desarrollo local y la democracia participativa a través de la integración de la comunidad al quehacer político. 


\section{GÉNERO E IDENTIDAD}

Lagarde (1999), manifiesta que los espacios de toma de decisiones siguen en poder de los hombres y de los sistemas patriarcales.

Méndez (2007), Pisquily (2007) y Lagarde (1999) argumentan que la participación de la mujer ha ido cambiando. En la actualidad, estas se han integrado en múltiples espacios (comunal, regional, nacional e internacional) desde la marginalidad democrática.

Sin embargo, aunque la mujer indígena se ha insertado en estos ámbitos, su participación es bastante limitada, debido a los sistemas patriarcales, a su condición económica, étnica, y de género.

La Procuraduría de la República de Nicaragua (2001), hace mención que el alcanzar espacios de poder para las mujeres en sociedades como la nuestra es un proceso lento y prolongado.

Htum, León \& Holgin citado por CEIMM-URACCAN (2010, p.9) plantea que la presencia de mujeres en ámbitos públicos no genera cambios automáticos, sino que da inicio a procesos formativos de largo plazo, que requieren tiempo y acompañamiento para el fortalecimiento de los liderazgos femeninos y el cambio de la cultura política de la población.

A partir de 1910, esta situación empieza a cambiar cuando se reconoce en el ámbito internacional los derechos y los aportes de la mujer a la sociedad, al reconocimiento del rol de la mujer en la transformación de las relaciones de género en función de un futuro más integrador (Lagarde, 2001).

Dixon \& McCoy (2010), en el artículo Participación Política y Liderazgo de las Mujeres Indígenas en América Latina: Caso Nicaragua, argumentan que entre los factores que inciden en la participación de las mujeres indígenas en los ámbitos de poder y administración pública están la pobreza extrema en zonas rurales, difícil acceso a los servicios, los tabúes culturales, creencias y costumbres, grado de exclusión social y desconocimiento de las leyes, entre otros.

Lagarde (1999), manifiesta que la política es un recurso de empoderamiento de las mujeres, y algunas mujeres no aceptan que otras tengan poderes. Esto se da especialmente cuando las mujeres han sido fundadoras en condiciones difíciles y han obtenido estatus y prestigio. 


\section{Materiales y métodos}

Esta es una investigación cualitativa, el abordaje fue a través del estudio de caso. Se enfatiza en el estudio etnográfico, por ser descriptiva. Destaca los valores, creencias, motivaciones, anhelos, formas de conductas y formas de interacción social de las mujeres indígenas de la comunidad de Rama Cay.

Las técnicas para la recopilación de la información fueron entrevistas semiestructuradas, dirigidas a mujeres y hombres líderes, adultos y jóvenes, asimismo se aplicó la observación participante. Estas entrevistas se realizaron desde y con el pueblo de Rama Cay.

El resultado se plasmó en una matriz donde se hizo referencia a los objetivos, preguntas y variables de estudio (cambio de roles, motivación, factores de participación, desafíos, limitantes y percepción). Este proceso visibilizó los sentimientos de las mujeres ramas en su interés de optar a cargos de liderazgo, pero también el porqué no se les permite optar a estas estructuras, aun teniendo la capacidad y conocimiento. Se identificaron los espacios de toma de decisión dentro del seno familiar, religioso, político y social. La articulación de la observación y las entrevistas permitieron lograr la información necesaria para darle salida a cada uno de los objetivos planteados.

La distribución fue de la siguiente manera:

- 16 adultos (10 mujeres y 6 hombres, entre 30 y 75 años de edad).

- 3 jóvenes (2 mujeres y 1 hombre, entre 18 y 25 años).

Las entrevistas se realizaron en lenguaje kriol para facilitar y obtener la información requerida.

La muestra se realizó con las mujeres líderes de la comunidad, aquellas que tuvieron la oportunidad de participar en espacios de la toma de decisiones. De igual manera se entrevistaron a hombres líderes para conocer su percepción y actitud hacia la mujer, Asimismo las diferentes percepciones de las personas de la comunidad con respecto a la participación en los espacios públicos. 


\section{Resultado y discusión}

Participación de la mujer rama en la comunidad de de Rama Cay en los espacios sociopolíticos de la toma de decisiones.

En la actualidad, las mujeres ramas han ido ganando espacio en la sociedad. A pesar de las adversidades, ellas participan en los ámbitos socio-políticos, en un consorcio patriarcal; con poca oportunidad pero con el deseo de formar parte integral de esos espacios.

Mujeres ramas de la comunidad de Rama Cay en cargos directivos

A continuación los cuadros números 1, 2 y 3, los cuales contienen información sobre la participación de las mujeres Indígenas ramas de la comunidad de Rama Cay, en los espacios de toma de decisión.

En el cuadro No. 1 se observa que sólo una mujer estuvo en la Junta Directiva de la comunidad en el 2003 fungiendo de tesorera, considerado un cargo secundario que normalmente se le asigna a la mujer por su capacidad y responsabilidad, los roles tradicionales de la toma de decisiones la mantienen los hombres.

Cuadro No. 1. Lista de autoridades comunales, período 2003

\begin{tabular}{|c|c|}
\hline \multicolumn{2}{|c|}{ Autoridades comunales } \\
\hline Carlos John Omier & Presidente \\
\hline Abelardo McCrea & 1er. Secretario \\
\hline Sebastián McCrea & 1er. secretario \\
\hline Basilio Benjamín & 2do secretario \\
\hline Ventura Hodgson & Tesorera \\
\hline Rufino Omier & Fiscal \\
\hline Antonio Alvárez & Vocal \\
\hline
\end{tabular}

Fuente: Consejo Regional RAAS (2010).

En el cuadro No. 2., se destaca la proyección de las mujeres en cargos administrativos de la comunidad en el 2006. Es por primera vez, que una mujer fue presidenta del gobierno comunal, lo cual no se ha repetido posteriormente al 2006. Es significativo que el $43 \%$ de las autoridades comunales para este período se constituyera por mujeres. Es además, un reflejo de la necesidad en cuanto a la generación de cambios en el liderazgo y desarrollo comunitario, lo cual implica una mayor visibilización de las capacidades de liderazgo de las mujeres. 
Cuadro No. 2. Lista de autoridades comunales período 2006

\begin{tabular}{|c|c|}
\hline \multicolumn{2}{|c|}{ Autoridades comunales } \\
\hline Hortensia Hernández & Presidenta \\
\hline Cristina Espinoza & 2da secretaria \\
\hline Mirna Daniel & Tesorera \\
\hline Jesús McCrea & Vicepresidente \\
\hline Miguel Ruiz & 1er. Secretario \\
\hline Agusto Daniel & Fiscal \\
\hline Antonio Álvarez & 2do vocal \\
\hline Sebastián Mc Crea & Miembro \\
\hline
\end{tabular}

Fuente: Consejo Regional RAAS (2010).

Los resultados del cuadro No. 3 reflejan un 44\% de mujeres en espacios de poder. Logran estar en los gobiernos comunales en cargos secundarios al igual que el período 2003. A pesar de estar en cargos secundarios, hay una mayor visibilidad en cuanto a cantidad de mujeres en cargos de presidente y segunda secretaria, tesorera y vocal.

Cuadro No. 3. Lista de autoridades comunales, 2010

\begin{tabular}{|c|c|}
\hline \multicolumn{2}{|c|}{ Autoridades comunales } \\
\hline Vicente Ruiz & Presidente \\
\hline Clevland McCrea & Vicepresidente \\
\hline Bety McCrea & Secretaria \\
\hline Meybell Martínez & Segunda secretaria \\
\hline Sonia Omier & Tesorera \\
\hline Santos Hernández & 2do fiscal \\
\hline Marcelo Daniel & Fiscal \\
\hline Fortilia Álvarez & Vocal \\
\hline Mateo McCrea & Vocal \\
\hline
\end{tabular}

Fuente: GTR-K (2010).

Considerando los resultados de los cuadros números 1 y 3 , se retomó lo planteado por Lagarde (1999), quien manifiesta que los espacios de la toma de decisiones siguen en poder de los sistemas patriarcales, restringiendo la participación de las mujeres a estos ámbitos.

El cuadro No. 2 (2006), refleja un cambio en la participación de la mujer a como mencionan: Méndez (2007), Pisquily (2007) y Lagarde (1999). Esto se menciona puesto que en la actualidad estas se han integrado en múltiples espacios a nivel comunal, regional, nacional e internacional desde la marginalidad democrática. 
Se enfatiza que en el 2006 las mujeres estaban en cargos de la toma de decisiones con una participación activa, lo cual difiere para este período según Pisquily (2007), al mencionar que a pesar de que la mujer indígena se ha insertado en estos ámbitos, su participación es bastante limitada, debido a los sistemas patriarcales, condición económica, étnica y de género. Sin embargo, esta contradicción sólo aplica para este período.

El Cuadro No. 4, demuestra la participación de dos mujeres ramas en espacios de poder en un período de 20 años. Se debe considerar que el pueblo rama es una población minoritaria y que reduce más la posibilidad de participación de las mujeres. En este marco, las mujeres ramas entrevistadas señalaron que todavía no han logrado escalar y tener una participación más activa en los Gobiernos Regionales, ni municipales, con una limitada presencia en los espacios de la toma de decisiones.

Cuadro No 4. Lista de las Concejales Regionales Ramas, 1990-2010

\begin{tabular}{|c|c|c|}
\hline \multicolumn{3}{|c|}{ Autoridades Regionales } \\
\hline Nombre & Cargo & Período \\
\hline Martina Thomas & Concejal Regional & 1990 \\
\hline Agnis Thomas & Concejal Regional & 2006 \\
\hline
\end{tabular}

Fuente: mujeres informante claves del estudio (2010).

Se muestra la inserción paulatina de la mujer en el cuadro No. 4, en los espacios de la toma de decisiones a partir de los años noventa, interconectando esto con la llegada de las Organizaciones no Gubernamentales como Acción Médica Cristiana, las universidades comunitarias (URACCAN y BICU).

La participación de las mujeres en el ámbito comunal ha sido notoria (Cuadros números 1, 2 y 3), al insertarse estas en las estructuras de gobierno, así como en la Comisión de Salud, Comisión de Educación, Centro de Mediación, entre otras. De igual manera, al participar en calidad de secretaria, vocal y presidenta del Gobierno Comunal (Cuadros 1, 2 у 3 ).

\section{Cambio de roles}

De acuerdo a quienes se entrevistó, ha surgido un cambio paulatino en los roles de la mujer rama, quien a pesar de jugar el papel de madre y esposa, ha ido cambiando ese patrón cultural y social que siempre la ha caracterizado; pasando a ser lideresas e insertándose en los ámbitos de administración pública. Este paso trascendental en la vida de las mujeres ramas es favorecido por las leyes internacionales y nacionales, que promueven el respeto y el derecho de las mujeres, con las oportunidades de acceder a la educación. Estas comienzan a prepararse como: profesoras, enfermeras, licenciadas en sociología, promotoras comunitarias, abogadas, e inician a realizar 
gestiones para el beneficio de sus comunidades. A partir de la formación integral y el empoderamiento inciden en estos cambios, forman parte de las transformaciones sociales y la gobernanza de su comunidad.

De igual manera, los resultados muestran que la participación de las mujeres en capacitaciones, talleres, foros y diplomados han sido la base fundamental para que ellas hayan adquirido los conocimientos sobre leyes, convenios y ratificaciones como la Convención sobre la eliminación de todas las formas de discriminación contra la mujer (CEDAW) aprobado en 1979 y el Convenio 169 de la OIT.

Con referencia a lo mencionado, una líder ha señalado:"Las mujeres tenemos la capacidad de liderazgo y hemos pasado de un estado a otro, porque pertenecemos a los gobiernos comunales, Consejo Regional".

$\mathrm{Al}$ respecto Dixon y McCoy (2010) argumentan que los avances en la legislación internacional y nacional, así como la presencia y acompañamiento de la URACCAN han contribuido e incidido en los cambios de roles en las mujeres. Las mujeres han ganado espacios participativos, y a través de los mismos cambiar sus roles a uno más integral, más justo y participando en espacios de toma de decisión en la comunidad.

De igual manera, Lagarde (2000) manifiesta que el rol de las mujeres en la transformación de las relaciones de género se da a partir de la promulgación de los convenios y leyes internacionales.

Las transformaciones sociales corresponden a la actualidad de los cambios en el mundo, relacionados a la búsqueda de una participación democrática, de equidad, a fin de lograr la construcción de una sociedad incluyente y más justa.

La presencia de las mujeres en el ámbito público es creciente, situación que responde a los cambios en las relaciones de género producto de procesos históricos y de la lucha de las mujeres por ser respetadas, hacer valer sus derechos y mejorar su calidad de vida.

Es evidente que las mujeres indígenas de la comunidad de Rama Cay están jugando un triple rol, el de madre, esposa y líder, aportando al desarrollo de su comunidad a pesar que son espacios de toma de decisión limitados..

En la comunidad se puede observar y analizar que no es fácil para ellas tener que jugar todos estos roles, porque primero tienen que cuidar a sus hijos, atender al marido, trabajar de cerca con las instituciones, empoderarse de las leyes, convenios y decretos para desempeñar un buen trabajo en la comunidad. 
Los cambios relacionados a la inclusión social de las mujeres ramas de la comunidad de Rama Cay en los ámbitos de poder responden a procesos lentos de formación y empoderamiento, que se generan en pro de los derechos de las mujeres.

Así mismo, las mujeres ramas también están en esos procesos de transformación social donde se generan esos espacios de toma de la decisión a nivel comunal, territorial y regional, todo eso ha sido posible con las capacitaciones promovidas por algunas instituciones.

\section{De las motivaciones de las mujeres}

Al preguntar qué fue lo que las motivó a insertarse en los ámbitos de poder, la mayoría de las entrevistadas señalaron lo siguiente: el deseo de contribuir a la lucha contra la violencia intrafamiliar; la lucha contra la discriminación por ser mujeres, por ser indígenas y la desigualdad de género. Así mismo, expresaron que por las oportunidades que representa tanto a nivel individual como familiar, para mejorar su calidad de vida, el deseo de apoyar a aquellas mujeres que aún continúan apáticas, a fin de incidir para que estas se apropien de las leyes y se empoderen.

Lo anterior coincide con lo planteado por la URACCAN (2009); Gall et al (2005) y Dixon \& McCoy (2010) en relación a las motivaciones de integración de las mujeres en los espacios de toma de decisiones. Estos autores argumentan que tanto hombres y mujeres tienen el derecho de optar por una educación integral, de aportar al desarrollo con identidad de sus comunidades y territorios, de disfrutar de una mejor calidad de vida. Además, este aporte a la construcción de una sociedad democrática y justa, debe realizarse en consideración al género: mujer, hombre, joven y adultos.

Entre las razones mencionadas por las entrevistadas también figuran el haber sido capacitadas por Acción Medica Cristiana y por la URACCAN a través del Centro de Estudios e Investigaciones de la Mujer Multiétnica (CEIMM); asimismo, contar con conocimiento de los derechos, las leyes, convenios internacionales y nacionales que promueven el respeto y la reivindicación de los derechos de las mujeres indígenas, lo cual las hizo reflexionar e incidió en su integración en estos espacios para aportar con sus ideas a la construcción de una sociedad justa.

Una mujer rama al hacer referencia al cambio de rol manifiesta: "Lo que me motivó es que soy humana y que tengo la misma capacidad que el hombre, tengo en alto mi autoestima, sé que soy alguien pensante igual que los hombres".

\section{Mujer líder de la comunidad}

Las entrevistadas también manifestaron que fueron motivadas por otras mujeres de su comunidad que han tenido experiencia en estos ámbitos de poder. 


\section{Factores que inciden en la participación efectiva de la mujer en los ámbitos administrativos y públicos}

Los resultados muestran que las mujeres indígenas de la comunidad de Rama Cay se enfrentan a un sin número de limitantes para acceder a los ámbitos de poder.

\section{Limitantes más sentidas}

Algunos hombres de la comunidad aún no valoran las capacidades y habilidades de liderazgo y de gestión que tienen las mujeres, lo cual incide en el acceso de sus esposas e hijas a la educación y la integración de estas en actividades de desarrollo de la comunidad, y por consiguiente en los espacios de toma de decisión.

$\mathrm{Al}$ respecto, un líder de la comunidad manifiesta que: "Una de las limitantes es que están en un mundo de hombres donde él es dueño, ellas sólo son parte del hogar”.

\section{Hombre líder de la comunidad}

Con referencia a lo anterior una comunitaria señala:

Uno de los retos que enfrenta muchas veces la mujer, es que sus maridos les impiden entrar en esos espacios. Tengo un ejemplo: mi prima iba a tomar una posición en la comunidad y su marido le dijo que no, y ella no pudo aceptarlo, ella pensó que podría coordinar un grupo de mujeres, que tenía la capacidad porque ella ya había concluido la secundaria. En la actualidad él no la deja estudiar.

\section{Comunitaria de Rama Cay}

Lo anterior coincide con lo planteado por otra de las comunitarias, la cual menciona lo siguiente: "Algunos hombres dicen que estamos locas, que perdemos el tiempo".

Otras de las mujeres entrevistadas expresan: "Los hombres dicen que las mujeres quieren ser líderes y tenerlos bajo sus pies, es por eso que quieren estar en esos espacios".

Otra de las limitantes señaladas por las entrevistadas fue el de la incidencia de las creencias y costumbres culturales, en cuanto a la presencia y acceso de las mujeres a los ámbitos públicos. El sólo hecho de ser mujeres solteras, y ser menores de 30 años representa un obstáculo para participar en estos espacios. La misma influencia de una sociedad patriarcal que se impone de generación a generación, es una de las limitantes. 


\section{Conclusiones}

La participación de las mujeres indígenas ramas en los espacios de toma de decisión constituyen una parte fundamental dentro de la comunidad, razón por la cual el modelo de desarrollo de la comunidad de Rama Cay debe ser integral, en donde se dé más espacio a las mujeres para constituir y participar en las esfera de poder dentro y fuera de la comunidad.

Así mismo, que ecuánimente se contemple la participación de las mujeres como uno de los ejes primordiales dentro de los procesos de cambio y de transformación social. Estos espacios no se les concede a la mujeres, sino que ellas tienen que ir forjándose metas como toda mujer emprendedora, luchadora, trascendiendo más allá de la cultura que la rodea.

A su vez, se han ido educando para poder entrar en espacios de toma de decisión participando activamente en reuniones, talleres, diplomados, intercambios con otras mujeres, todo esto es debido a las leyes, convenios, decretos internacionales y sobre todo el empoderamiento por parte de ellas para poder contribuir al desarrollo de su comunidad. También, han sobresalido en las actividades de la comunidad al ser más participativas y visibles dentro de los espacios de toma de decisiones.

Debido al contexto en que se han desarrollado, ellas han tenido que estudiar y aprender otras lenguas, necesidad para poder comunicarse con otras personas. Una de sus limitantes es la expresión oral, la comunicación fluida con otras personas fuera de la comunidad.

Pero a pesar de los obstáculos que han tenido que solventar, las mujeres indígenas ramas que han estado inmersas en los ámbitos públicos dentro y fuera de la comunidad, son un ejemplo vivo para mujeres indígenas de otras comunidades que todavía permanecen bajo el dominio patriarcal. Y, es dentro de las esferas de este tipo de poder que ha logrado cambios en los patrones culturales y su cosmovisión como mujer indígena.

De igual manera se tenía la necesidad de conocer cuál era el rol que jugaban las mujeres en la comunidad, cuáles eran los retos, debilidades, limitantes que enfrentaban durante su vida diaria para entender cuál es la situación de la mujer con relación a su participación en los espacios públicos.

Después de haber compartido con las personas comunitarias, se pudo comprender que el papel que juegan las mujeres ramas es más que el de ser madre y esposa abnegada que cuida de su hogar y de sus hijos, y que toma muy poca decisión dentro del hogar. Ellas también juegan el rol de lideresas al participar en los gobiernos comunales, territoriales y regionales con muy poca presencia. Las mujeres ramas 
mencionan que fue desde los noventa desde cuando se manifestaron en contra de las desigualdades de género.

Algunas de las limitantes que enfrentan las mujeres ramas en su comunidad son la exclusión social por parte de los hombres de la comunidad y por la sociedad en general. Por ser mujer muchas veces se les impedía seguir estudiando, pero a pesar de esas dificultades se han insertado y son más visibles ante la Sociedad Civil y a nivel regional.

Los diferentes roles ha desempeñar como mujer son difíciles, porque la misma sociedad las cohíbe, negándoles la oportunidad de participar en esos espacios de toma de decisiones, teniendo que confrontar al hombre para entrar en esos espacios de poder.

La poca presencia y participación de las mujeres indígenas ramas de Rama Cay en los espacios de toma de decisiones, responde al desconocimiento de las leyes y de la apropiación del enfoque de género. Es por eso que el empoderamiento en leyes permite que las mujeres, en especial las mujeres ramas, participen más, logrando su integración en los espacios de toma de decisión.

\section{Recomendaciones}

\section{A líderes comunales y territoriales:}

- Analizar con los comunitarios y comunitarias los resultados de esta investigación para que valoren como están organizados en la comunidad y cómo es la participación de las mujeres.

- Brindar más espacios de participación activa a las mujeres indígenas ramas en las estructuras de los gobiernos comunales y gobiernos territoriales.

- Promover el respeto y reconocimiento de las capacidades de gestión de las mujeres de la comunidad.

\section{A las mujeres líderes:}

- Continuar participando en procesos de formación afín de obtener mayor conocimiento sobre la legislación, la promoción del respeto, participación y reivindicaciones de sus derechos.

- Formar alianzas con hombres y líderes afín de contribuir conjuntamente al desarrollo de la comunidad.

- Continuar incidiendo ante las autoridades comunales y territoriales afín de lograr la plena participación de la mujer en los ámbitos de poder. 


\section{A las ONG 's y universidades:}

- Continuar acompañando, asesorando, fortaleciendo las capacidades y empoderando a las mujeres indígenas de Rama Cay, a fin de lograr una mayor integración y participación cualitativa en los ámbitos públicos y administrativos.

- Promover programas de sensibilización dirigidos a la sociedad civil, sobre el valor de las mujeres y la importancia de la participación de estas en los ámbitos de poder.

\section{AI MINED}

- Integrar en el currículo educativo la temática de género afín concientizar a los niños y jóvenes sobre la importancia y el valor de la mujer. Promover el respeto y la plena participación de los hombres y las mujeres en la construcción de una sociedad más integradora.

\section{Al Gobierno Regional /Secretaría de la Mujer}

- Promover la institucionalización del enfoque y políticas de género a fin de lograr una mayor integración de las mujeres en los ámbitos públicos.

- Fomentar el respeto y reconocimiento de los derechos de igualdad y de participación de la mujer.

- Aplicar la política de género que aprobaron en años recientes.

\section{Lista de Referencia}

CEIMM-URACCAN (2009). Política Intercultural de Género. Documento no publicado. Managua, Nicaragua.

Gutiérrez et al. (2009). Aporte de la Mujer Rama al Desarrollo Identitario de las Comunidades de Rama Cay y la Zompopera, Bluefields Ciencia e Interculturalidad: Revista para el Diálogo Intercientífico e Intercultural. Edición 4,

Lagarde (1996). Género feminismo (Desarrollo humana y Democracia).

Lagarde (2000). Claves Feministas para liderazgos entrañables. 1 ed. Managua: Puntos de Encuentro. 93 p.

Mejía et al. (2003).Tras las vetas de la investigación cualitativa. Perspectiva y acercamientos desde la práctica. México. 